\title{
Saber tradicional, ética de la tierra y sustentabilidad
}

\author{
THOMAS HEYD
}

University of Victoria, Canadá

\begin{abstract}
RESUMEN. Éste es el momento crucial para emprender formas de vida sustentables, por lo que resulta esencial adoptar una ética de la tierra que reconozca el valor intrínseco además del valor útil de los seres que pueblan nuestro medio ambiente natural. En este ensayo empiezo enfocando los obstáculos a que se enfrenta el desarrollo de una ética de la tierra, obstáculos que resultan ser el producto de conocimientos limitados y suposiciones equivocadas. En resolución de esas problemáticas prácticas apunto, primero, la existencia de inclinaciones naturales hacia la tierra y la vida. Además propongo que en el saber tradicional de los pueblos que han convivido mucho tiempo con sus espacios tenemos modelos de formas del conocer que nos pueden servir de modelo para llegar a desarrollar una cultura de aprecio de la tierra y de sus habitantes.
\end{abstract}

\section{INTRODUCCIÓN}

A medida que aumenta la velocidad del desarrollo global también aumenta con urgencia la necesidad de desarrollar formas de vida que sean durables o sustentables ${ }^{1}$. Aun si el significado del término «sustentabilidad» está en disputa, voy a
ABSTRACT. This is the crucial moment to take on sustentable forms of life, and hence it is now of primary importance to adopt a land ethic that takes into account not only the use value but also the intrinsic value of the beings that make up our natural environment. In this essay I begin by focusing on the obstacles that the development of a land ethic has to face, obstacles that are due to limitations in knowledge and to mistaken assumptions. As a way to resolve these practical difficulties I first point out the existence of our natural inclinations toward the land and life. Furthermore, I propose that the traditional knowledge of those peoples that have lived for extended periods with their environments can serve us as models for the development of a culture of appreciation for the earth and its inhabitants.

suponer que representa el tipo de actividad que es compatible con el bienestar de los seres humanos actuales al tiempo que con el de los futuros ${ }^{2}$, en ecosistemas viables. Entiendo por ecosistemas viables ecosistemas que se desarrollan de forma normal, sin pérdidas de especies $\mathrm{y}$ de hábitat importantes a causa de la intervención huma- 
na. Propongo que la ética de la tierra propuesta por el ingeniero forestal y pensador ecologista Aldo Leopold constituya una forma reseñable de concebir precisamente tal forma de vida sustentable.

La cuestión crucial es cómo podemos llegar a adoptar tal ética de la tierra dados los obstáculos a que se enfrenta. En parte, la respuesta consiste en que tenemos que darnos cuenta de nuestras inclinaciones naturales hacia la tierra y la vida (discutidas bajo los términos topofilia y biofilia). Otro ingrediente crucial para que se adopte una ética de la tierra, como la concibe Leopold, consiste en el aprendizaje de algunas de las formas del conocer que son características del saber tradicional (lo que en inglés se llama indigenous knowledge y tiene afinidad con el llamado «saber local»).

A continuación comienzo con una clarificación de la ética de la tierra de Leopold, y de los obstáculos al desarrollo de tal ética. Después de tomar nota del papel a jugar por la topofilia y la biofilia, prosigo con una explicación de cómo las formas del conocimiento que generan el saber tradicional pueden servir como condiciones importantes para traspasar los obstáculos señalados por Leopold. De paso considero una importante objeción a mi propósito que resulta de la tensión entre enunciados fácticos y enunciados normativos. Acabo concluyendo que las formas del conocimiento tradicionales pueden hacer una contribución muy importante al desarrollo de una ética que apoya la sustentabilidad y, por lo tanto, al desarrollo de una ética de la tierra.

\section{La ética de la tierra}

Aldo Leopold invocó la adopción de una ética de la tierra como reacción a la falta de conciencia entre sus contemporáneos de la frecuentemente irreversible degradación de «la tierra, y los animales y plantas que viven sobre ella ${ }^{3} \gg(74)$. Entre filósofos se continúa debatiendo si el buen trato de los seres no humanos requiere un nuevo tipo de teoría de ética integral que considera a los seres individuales meramente en cuanto son miembros de comunidades (lo que en inglés se ha llamado holism) o, al contrario, si simplemente se requiere una extensión de las teorías éticas individualistas ya existentes ${ }^{4}$. Lo que hay que tener en cuenta es que el debate se enfoca en cómo justificar un respeto a la naturaleza no humana.

Aunque generalmente se le ubica a Leopold entre los que abogan por la necesidad de una nueva teoría de ética integral enfocada en comunidades, se puede argumentar que en realidad él se preocupó menos por una nueva justificación del respeto por el medio ambiente natural que por el desarrollo de una nueva voluntad de actuar que exprese respeto por la tierra y su vida. Se podría decir que la demanda de Leopold por una ética de la tierra principalmente es una demanda por la adopción de un nuevo ethos o nueva conciencia de nuestra responsabilidad respecto a nuestro medio ambiente natural. Es decir, lo que Leopold considera necesario es una nueva forma de vida adecuada al valor que, en nuestros momentos más lúcidos, pretendemos reconocer en la tierra y sus habitantes.

Leopold concibió la relación entre los seres humanos y el medio ambiente natural en términos de la participación en una «comunidad biótica" que comprende suelos, aguas, animales y plantas. Ésta es una concepción ecológica del ser humano en su ambiente; es una propuesta que cabe dentro de una ecología humana o antropología ecológica. Leopold supuso que, aunque «la alteración, la gestión y el uso de estos «recursos» es inevitable, el respeto por el valor de los miembros no humanos de la comunidad biótica conllevaría la afirmación de «su derecho a una existencia continuada y, por lo menos en algunos lugares, [su derecho a] una existencia continuada en un estado natural» (74). 
Quizás resulte curioso, pero Leopold acabó incrédulo respecto a la suposición que «un aumento en educación para la conservación» llevaría a las deseadas prácticas ecológicamente apropiadas. Tal como él lo veía, la educación para la conservación que se les proponía a sus contemporáneos se reducía poco menos que al siguiente imperativo: «obedece a la ley, vota correctamente, hazte miembro de algunas organizaciones, y practica en tu tierra cuanta conservación te sea provechosa; el gobierno hará el resto» (75). Para Leopold tal educación en conservación ambiental no podía detener la marea de destrucción medioambiental y no llevaría a generar una ética de la tierra, porque se olvidaba de observar que se requiere más que un egocentrismo iluminado, o sea, más que un interés en el propio bienestar a corto plazo.

Leopold nos ilustra las limitaciones de este tipo de «egocentrismo iluminado» señalando que, cuando se proponen políticas basadas en esta actitud, los resultados son bastante insatisfactorios. Recuerda que cuando intentaron contravenir a la erosión de las tierras en el Estado de Wisconsin de USA en los años 1930 se propusieron dos métodos convergentes: La educación en conservación ambiental y la auto-regulación. «Los agricultores», sin embargo, «sólo seleccionaban esas prácticas de restauración que de todos modos les eran provechosas, pero ignoraban las que beneficiaban a la comunidad y no directamente a ellos mismos». Leopold concluye que «el resultado neto fue que tenemos más educación, pero menos tierra, menos bosques en buen estado de salud, y tantas inundaciones como antes» (76). En contraste, la educación en conservación ambiental efectiva cultivaría la conciencia ecológica, es decir, una voluntad de actuar que refleja «una convicción de responsabilidad personal por la salud de la tierra» (80-81).

Aunque hoy día es común manifestarse a favor de la protección de un medio ambiente natural sano y a favor del desarrollo sustentable, la opinión predominante todavía tiende a ser que los individuos, bien sea como particulares, bien como propietarios de acciones bursátiles, bien como personas que trabajan por empresas industriales, no tienen otro sentimiento de responsabilidad que hacia sí mismos y sus familiares. Ahora bien, afortunadamente la gestión que favorece al bienestar del medio ambiente natural a veces concuerda con el interés propio de los dueños y usuarios de las tierras, pero cuando éste no es el caso hay pocas personas que perciben una obligación o una responsabilidad que les impulse a proteger la integridad del medio ambiente natural. Por ejemplo, pese a que los bosques de árboles centenarios pueden proveer de importantes nichos a una variedad de plantas y animales en riesgo de extinción, en la Costa Oeste de Canadá se sigue dando prioridad a la desenfrenada tala rasa de los bosques por parte de las grandes empresas ${ }^{5}$. (Y esto a pesar de que estas grandes empresas reemplazan cada vez más trabajos sustentables por máquinas.) Evidentemente el tipo de conciencia y sentimiento de responsabilidad por el bienestar de la tierra y de sus habitantes, descrito por Leopold cuando hablaba de la ética de la tierra, aún no ha echado raíces en muchos lugares.

\section{Obstáculos a la ética de la tierra}

Leopold sugirió que hay barreras específicas al desarrollo de la ética de la tierra. «Es inconcebible», dijo Leopold, «que haya una relación ética con la tierra sin amor, respeto y admiración por la tierra, y una alta consideración por su valor» (82). Desde su perspectiva tal relación ética con la tierra está socavada por tres factores principales: Nuestro creciente aislamiento de los procesos naturales, nuestra percepción de tener una relación adversa con la tierra, y nuestra creencia en la «falacia» 
del determinismo económico. Trato estos obstáculos en el orden apuntado aquí.

Primero, Leopold tomó nota de que «nuestro sistema educativo y económico se está alejando y no se está acercando a una concienciación intensa por la tierra» (82). Señala que la orientación hacia los artefactos de nuestras sociedades bloquean el conocimiento directo del medio ambiente natural del cual dependemos en realidad para nuestro sustento. En los casos extremos esto se demuestra en nuestra preferencia por una multiplicidad de «sustitutos sintéticos» (82). Para ilustrar esto, Leopold señaló el hecho que para mucha gente la tierra no tiene interés a menos que les sea promocionada como «campo de golf o como "zona escénica"» (82).

Desde los tiempos de Leopold, en las primeras décadas del siglo Xx la sustitución de lo natural por lo artificial se ha acelerado repetidas veces. Este proceso ha avanzado hasta incluir actividades que anteriormente habrían sido descritas como propias de los entusiastas de la naturaleza pura. El esquí y la escalada, por ejemplo, se están volviendo cada vez más actividades «sintéticas», diseñadas en su totalidad por los ingenieros; la nieve producida artificialmente, como en el nuevo centro cerca de Madrid, y las paredes para escalar hechas en cemento armado, están haciéndose preferibles a sus equivalentes naturales ${ }^{6}$. Además, después de medio centenario de divulgación de una cierta pseudo-realidad sobre las pantallas de televisión en nuestros hogares, aparentemente ya pronto vamos a vivir la diseminación en masa de máquinas que generarán un sinfín de experiencias sintéticas: a saber, las realidades virtuales ${ }^{7}$. Esta alienación creciente de la tierra y de sus procesos vitales va en contra del desarrollo de una ética de la tierra. El desarrollo de tal ética requiere una importante reforma de nuestros sistemas educativos y económicos; requiere un esfuerzo consciente y concertado para re-conocer nuestro enraizamiento en el medio ambiente natural ${ }^{8}$.
El segundo obstáculo al desarrollo de una ética de la tierra señalado por Leopold es la concepción de la tierra como un adversario al que tenemos que forzar a que nos dé para vivir. Esta concepción contribuye a que nos sintamos alienados de la tierra. Si la tierra es vista como «un amo que nos mantiene en esclavitud», entonces el reemplazamiento de los bosques naturales por bosques de plantación, la minería indiscriminada aun en regiones silvestres, y el amplio uso de pesticidas y herbicidas en campos y bosques se vuelve enteramente justificado y aun deseable. En respuesta a esta dificultad Leopold manifiesta que urge la diseminación del conocimiento genuinamente ecológico sobre la tierra y sobre nuestro lugar en el medio ambiente natural. Leopold propone que el reconocimiento de nuestra participación en la comunidad ecológica, que incluye a una diversidad de especies, nos llevará a adoptar el imperativo siguiente: «Una acción es correcta si tiende a preservar la integridad, la estabilidad, y la belleza de la comunidad biótica. Es incorrecta si tiende en sentido contrario» (82).

El tercer obstáculo al desarrollo de una ética de la tierra señalado por Leopold es la creencia de que necesariamente «los factores económicos determinan todos los usos de la tierra» (82). Aunque admita que los factores económicos sí pueden ocasionar una importante limitación a nuestra preocupación por el bienestar de la tierra como tal, Leopold indica que «simplemente no es verdad» que esos factores son, o tienen que ser, los únicos valores relevantes en las decisiones que afectan el uso de la tierra; más bien, «un sinnúmero de acciones y actitudes, que quizás comprendan la mayoría de las relaciones con la tierra, están determinadas por el gusto y las predilecciones del que usa la tierra y no por su billetera». (82) Lo que Leopold tiene en mente es que generalmente los dueños y usuarios de un terreno sí tienen opciones en su uso, y que no están confrontados a la 
quiebra simplemente por no proseguir el camino que maximiza los beneficios exclusivamente económicos. Por ejemplo, en la Isla de Vancouver hay personas que talan sus bosques seleccionando los árboles a cortar según criterios ecológicos. Podemos asumir que, según Leopold, la reflexión sobre nuestro papel como miembros de la comunidad biótica, compuesta por la tierra y sus habitantes, conllevaría nuestro reconocimiento del hecho de que los valores no económicos pueden y deben jugar un papel importante en nuestras decisiones respecto al uso de la tierra.

En síntesis: Leopold compara nuestras intervenciones en la tierra con la actitud quijotesca de reformar la Alhambra, ese exquisito apogeo de arquitectura nazarí del antiguo $\mathrm{Al}$ Andalus. Dice que «estamos remodelando la Alhambra con una excavadora y estamos orgullosos del camino que ya hemos abierto» (82). Leopold se dio cuenta que, a pesar de nuestro mucho hablar del valor intrínseco de la tierra y de su vida, seguimos destruyendo su complejo lienzo sin más. Sin embargo, pese a los obstáculos indicados, Leopold argüye que sí es posible adoptar una ética de la tierra que respete al medio ambiente natural por el valor que tiene de por sí. Lo que Leopold sugiere es una nueva forma de vida, un nuevo ethos que manifieste un compromiso práctico de actuar según ese respeto por la tierra que profesamos tener. Pero, ¿qué puede dar fundamento a esa nueva ética? Es decir, ¿en qué puede basarse tal nueva forma de vida?

\section{Topofilia, biofilia y saber tradicional}

Los obstáculos al desarrollo de la ética de la tierra descritos por Leopold, es decir, nuestro creciente aislamiento de los procesos naturales, la concepción de ser adversarios de la tierra, y la creencia en la falacia del determinismo económico, sugieren que por lo general no tenemos un genuino respeto por la tierra. No obstante, esta situación no es inevitable.

Hay razones para creer que somos biológicamente capaces de valorar positivamente los lugares naturales y los seres vivos que habitan en tales lugares, $y$ tal vez incluso estemos genéticamente predispuestos a este tipo de valoración. En apoyo de esta suposición pensemos en la ubicuidad de lo que se ha llamado la «topofilia» (o sea, el amor al topos o lugar) en las diversas culturas del mundo: según el importante estudio de $\mathrm{Yi}-\mathrm{Fu}$ Tuan, todos los pueblos del mundo tienen lazos afectivos con los lugares que conocen bien ${ }^{9}$. Similarmente, pensemos en la conclusión de los estudios del sociobiólogo E. O. Wilson que propone la realidad de la biofilia, esto es, que tenemos «una tendencia innata de enfocarnos en la vida y en los procesos similares a la vida ${ }^{10}$.

Sin adentrarnos mucho en el tema podemos constatar que es cierto que el grado de topofilia y de biofilia varía entre las personas y aun entre las culturas, pero para nuestra discusión la cuestión realmente importante más bien es cómo se puede activar este potencial por la valoración positiva de los lugares naturales y de la vida, para que se genere la conciencia por la integridad y por la salud de la tierra. Yo propongo que el desarrollo de la ética de la tierra depende crucialmente de las formas del conocimiento que, por ejemplo, se exhiben en lo que se ha llamado «saber tradicional» o «saber vernáculo».

El «saber tradicional» (denominado indigenous knowledge en inglés) recientemente ha recibido considerable atención académica e institucional ${ }^{11}$. El estudio, la aplicación y el registro del saber tradicional, considerado como una parte de las técnicas tradicionales o vernáculas para vivir en, y con, los ambientes naturales, se ha vuelto un campo de gran interés y valor para investigadores de las sociedades industrializadas, así como para los representantes de los pueblos autóctonos. 
El saber tradicional o vernáculo es una expresión cuyos límites conceptuales aún están en evolución. Se ha utilizado para hacer referencia al conocimiento de los pueblos «indígenas», que es un sinónimo aproximado de los términos «aborígenes», «nativos» o «autóctonos»; es decir, se ha utilizado para hacer referencia al conocimiento de las personas que son los descendientes de los habitantes originarios de un cierto territorio ${ }^{12}$. La expresión indigenous knowledge (saber tradicional) también se ha utilizado de una forma más general para hacer referencia al conocimiento «local» adquirido por personas que han pasado un tiempo importante en una localidad, sin que importe si sus antepasados tuvieron su origen en ese lugar ${ }^{13}$.

El saber tradicional puede ser explícito y verse expresado, por ejemplo, en las historias o en consejos transmitidos de generación en generación, pero también puede ser implícito y meramente presente en prácticas tradicionales específicas que determinan, por ejemplo, cuándo o dónde se puede pescar, y con cuales técnicas e ins- trumentos ${ }^{14}$

Lo más interesante para nuestra discusión es que el saber tradicional se ha reconocido 1) como enfocado primariamente sobre las calidades de las cosas y menos en su cuantificación, 2) que generalmente es el resultado de una relación directa y personal, y 3) que se ha acumulado diacrónicamente (es decir, que se ha obtenido como sedimento de la permanencia de un grupo de personas en un lugar a lo largo de extensos períodos de tiempo en una cierta localidad) ${ }^{15}$.

Más aún, en muchas ocasiones se ha documentado la conexión entre formas tradicionales del conocimiento y los valores pro-ambientales. Deborah Bird Rose ha documentado, por ejemplo, la manera en que los patrones del buen actuar, según la cultura de los aborígenes australianos yarralin, están basados en su milenario conocimiento, obtenido por la interacción directa, con las plantas, los animales y los lugares en que viven ${ }^{16}$. Rose propone que la particular forma del conocimiento de la tierra de los pueblos yarralin está completamente integrado con su práctica moral y su filosofía respecto a la tierra. Esto tiene efectos, por ejemplo, en sus prácticas y normas de qué animales se pueden cazar y cuándo.

Como es bien sabido, se ha observado a menudo que las sociedades tradicionales nos proveen de modelos excepcionales acerca de una relación con el mundo natural que muestra su respeto a los seres no humanos en sus papeles ecosistémicos. En Centroamérica y en Amazonia, por ejemplo, se practica una forma de agricultura que permite a la selva regenerarse continuamente. En muchas regiones del planeta existen prohibiciones estacionales de cazar o de recolectar ciertos productos naturales, permitiendo así su desarrollo sustentable. En resumen, mi tesis es que las formas tradicionales del conocimiento, es decir, las maneras autóctonas y locales de conceptualizar conjuntamente el mundo natural y el humano, pueden desempeñar un papel crucial en la generación de actitudes ecológicamente apropiadas de convivencia con la naturaleza.

Quiero anticipar una objeción, sin embargo, que tal vez sea ser presentada a mi tesis. Se puede argüir que estoy suponiendo, equivocadamente, que una moralidad adecuada, promotora de la sustentabilidad, puede ser derivada de las formas tradicionales del conocer.

\section{Relacionando la ética con el conocimiento}

El pensar ordinario y la filosofía ortodoxa suelen estar en radical desacuerdo respecto al papel de los enunciados fácticos en temas medioambientales. Se ha señalado que a menudo los que escriben periodísticamente sobre temas medioambientales se mueven libremente entre enunciados fácticos (respecto a la contaminación ambien- 
tal, por ejemplo) a enunciados normativos sobre acciones que debían realizarse desde el punto de vista ético (como sería la prevención o la mitigación de la contaminación ambiental). Estas propuestas han sido criticadas porque pasan por alto la distinción fundamental entre enunciados fácticos y enunciados normativos habida cuenta de que la ortodoxia filosófica, que se remonta hasta el ilustre David Hume, declara que enunciados normativos no pueden derivarse de enunciados fácticos. Por lo tanto, se señala que, a partir del hecho que existe contaminación medioambiental causada por nuestras industrias, no se puede deducir directamente que la actividad industrial que genera esta contaminación tiene que cesar. Esa conclusión sólo se puede derivar si se incluye en este razonamiento un enunciado normativo que representa una valoración adecuada, tal como puede ser la suposición que «la generación de contaminación ambiental dañina a los seres vivos no debe permitirse».

Sin embargo, hay razones para creer que la relación entre enunciados fácticos y enunciados normativos, esto es, entre el conocimiento y la ética, es más estrecha que lo que esta ortodoxia sugiere. Esto se pone de manifiesto si reflexionamos sobre los supuestos fundamentales de las ciencias naturales, las cuales representan el epítome del conocimiento objetivo en nuestras sociedades llamadas «occidentales». Se ha hecho cada vez más claro, por lo menos desde el trabajo de Thomas Kuhn sobre las revoluciones científicas, que las ciencias naturales no están libres de valores. Está haciéndose patente que, en gran parte, las ciencias están estructuradas por el fin de hacer posible que el mundo natural sea asequible a la predicción y a la manipulación por el control técnico. Desde este punto de vista, la «objetividad» de enunciados fácticos se traduce en que estos enunciados no delaten un compromiso con valores diferentes a los valores determinantes de la ciencia, que, como acabamos de resumir, son la reducción del mundo natural a la predicción, y a la manipulación y el control de los procesos naturales por los seres humanos. Esto es tanto como decir que un enunciado es considerado científicamente fáctico si no se halla comprometido por la retórica política o religiosa, por ejemplo. Pero advirtamos que esta limitación en sí también es la expresión de una valoración.

Por ejemplo, los conflictos sobre políticas que enfrentan opciones supuestamente basadas en razones «objetivas» a opciones basadas en razones supuestamente «subjetivas» frecuentemente dependen en forma decisiva de valores ocultos, pero subyacentes, en el punto de vista presuntamente «objetivo». Consideremos las disputas entre los que apelan al valor «objetivo» (determinado en forma cuantificada y en términos monetarios) que supuestamente tiene el desarrollo de ciertas tierras por medio de la tala rasa de los bosques, la minería o la construcción, y los que apelan al valor «subjetivo" (determinado en términos quizás estéticos o de experiencia personal) que esas mismas tierras tienen en un estado sin alteración, o sea, en su estado natural. Obviamente, estas determinaciones del valor «objetivo» de los bosques y de la tierra presuponen la previa disponibilidad de los terrenos en cuestión para su manipulación en términos de la tala de árboles, de la minería y de la construcción.

Nos podemos preguntar, sin embargo, qué es lo que justifica en un principio la suposición de su disponibilidad para estos fines; está claro que no todo lo que se puede realizar físicamente corresponde a 10 que se debe realizar desde un punto de vista del respeto por los seres vivos que nos rodean. Los valores ocultos y subyacentes a las evaluaciones supuestamente «objetivas» solamente se hacen patentes en los casos extremos, como, por ejemplo, cuando nos damos cuenta de lo inapropiado que sería evaluar a los seres humanos como meros recursos de órganos o de 
minerales según las leyes del mercado. Pero es precisamente este tipo de valoración implícita la que está siendo utilizada cuando se trata a los bosques y a las formaciones geológicas como meros yacimientos de papel higiénico o de materias primas para las industrias de productos desechables.

En fin, en respuesta a la objeción que hemos mencionado aclaro que no estoy argumentando que un enunciado fáctico pueda ser derivado de un enunciado normativo, pero que, hasta cierto punto, los enunciados fácticos ya presuponen ciertas valoraciones o enunciados normativos. Ahora bien, si las valoraciones o los enunciados normativos entran ineludiblemente en los enunciados fácticos, entonces, si nuestro objetivo es promocionar el desarrollo de la ética de la tierra, es imperativo que nos fijemos en las formas del conocimiento que sí están armonizadas con valoraciones que son congruentes con esa ética de la tierra.

\section{Conclusión}

Desde este momento la discusión se vuelve un tanto empírica, ya que la mejor manera de determinar formas del saber que son congruentes con la ética de la tierra, o sea, con una ética que promueve la sustentabilidad, consiste en localizar ejemplares del saber que manifiestan esos par- ticulares valores. En este ensayo supuse que las formas del conocimiento que dan lugar al tipo de saber tradicional, también llamado conocimiento local o indígena, tienden a ser especialmente congruentes con una ética de la tierra que promueve la sustentabilidad.

Ciertamente, es apropiado adquirir, en cuanto sea posible, y en cuanto se nos sea ofrecido por sus poseedores legítimos, el saber tradicional de las personas nativas de un lugar, para lograr la mejor comprensión posible de esos particulares lugares. Pero, para nuestros propósitos, es aún más importante que consideremos como modelos las formas del conocimiento ejemplificadas en esas sociedades. En otras palabras, mi propuesta es que adoptemos las formas del conocimiento de la tierra que atienden a las relaciones personales que desarrollamos con nuestro medio ambiente, que se focaliza en los muchos y variados recursos que ese entorno natural nos ofrece como obsequios, que favorece el cuidado cuando se proponen trastornos ecosistémicos, que tiene en cuenta el papel que el medio ambiente natural tiene en las historias personales de los habitantes de los lugares, etc. Creo que es posible que la adopción de tales formas del conocimiento sí puedan llevarnos a un nuevo cuidado por la tierra y sus habitantes, es decir, nos pueda llevar a una auténtica ét $i$ ca de la tierra ${ }^{17}$.

\footnotetext{
' El término «sustentable» es una traducción del término alemán «nachhaltig» que literalmente significa que una cosa «aguanta después» o que perdura. Desde que se lanzó el Reportaje Brundtland, que oficialmente se llamó Our Common Future, Nueva York, Oxford University Press, 1987, de la Comisión Mundial sobre Ambiente y Desarrollo y la Cumbre de la Tierra de Río del 1992 (La Conferencia de Naciones Unidas sobre el Ambiente y Desarrollo), ha habido una discusión bastante grande sobre el significado
}

que se debía dar a las expresiones «desarrollo sustentable» $\mathrm{y}$ «sustentabilidad». Ha habido interpretaciones muy divergentes de estos tếrminos, que van desde la suposición que la sustentabilidad requiere que no haya intervención humana alguna en la naturaleza hasta la idea que el desarrollo sustentable demanda que se aumente la actividad económica hasta cincuenta veces lo que eran las tasas de los años 1980

2 Esta definición la adopto del Reportaje de Brundtland. 
3 Todas las referencias a Aldo Leopold son de $\boldsymbol{A}$ Sand County Almanac with Other Essays on Conservation from Round River, Nueva York, Oxford University Press, 1966; la edición original se publicó en 1949, pp. 217-241. Incluyo las referencias a páginas citadas en el texto mismo.

4 Véase, por ejemplo, J. Baird Callicott, In Defen se of the Land Ethic, Albany, State University de Nueva York, 1989, y especialmente «Animal Liberation: a Triangular Affair», pp. 15-38, y «The Conceptual Foundations of the Land Ethic», pp. 75-99.

5 Se ha dado un ejemplo muy contundente en los años 90 en la Isla de Galiano, en la Costa Oeste de Canadá, donde se ha hecho una venta de terrenos con bosques primarios a los intereses de explotación forestal, a pesar de la protesta muy fuerte de los ciudadanos del lugar.

6 La noción de que la nieve natural es dispensable en las pistas de esquí hoy día ha sido argumentado con gran claridad por Albert Borgmann en una conferencia titulada «The Nature of Reality and the Reality of Nature», presentada en la University of Alberta, 1993.

7 En este contexto véase también la discusión de Albert Borgmann de la hiperrealidad en su Crossing the Postmodern Divide, Chicago, University of Chicago Press, 1992, pp. 82-97.

8 Véase también David Orr, «Love It or Lose It: the Coming Biophilia Revolution», Orion 13, Invierno 1994, pp. 8-15.

9 Yi-Fu Tuan, Topophilia: a Study of Environmental Perception, Attitudes and Values, Nueva York, E. O. Wilson, Biophilia: The Human Bond With Other Species, Cambridge, Harvard University Press, 1984, Prentice-Hall, 1974.

10 Wilson, Biophilia: The Human Bond with Other Species, Cambridge, Harvard University Press, 1984,
Prentice-Hall, 1974. Stephen R. Kellert y E. O. Wilson, The Biophilia Hypothesis, Washington D. C., Island Press, 1993.

11 Thomas Heyd, «Indigenous Knowledge, Emancipation and Alienation», Knowledge and Policy, 8 1994, pp. 63-73; D. M. Warren, D. Brokensha y L. J. Slikkerveer (eds.), Indigenous Knowledge Systems Londres, Kegan Paul, 1993; World Commission on Environment and Development 1987; Agenda 21 en J. Quarrie (ed.), Earth Summit, Londres, Regency Press, 1992.

12 Respecto a este perspectiva véase Agenda 21 , capítulo 26.

${ }^{13}$ E. Hunn, «What is Traditional Knowledge», en Williams y Baines (eds.), Traditional Ecological Knowledge, Canberra, Centre for Research and Environmental Studies, Australian National University, 1993, pp. 13-15.

14 H. T. Lewis, «Traditional Ecological Knowledge: Some Definitions», en Williams y Baines (eds.), Traditional Ecological Knowledge, pp. 8-12.

15 F. Berkes, «Traditional Ecological Knowledge in Perspective», en J. T. Inglis (ed.), Traditional Ecological Knowledge: Concepts and Cases, Ottawa, International Program on Traditional Ecological Knowledge, I.D.R.C., 1993, p. 40.

16 Deborah Bird Rose, «Exploring an Aboriginal Land Ethic», Meanjin, 47, 1988, pp. 378-387.

17 Le estoy muy agradecido a la Prof. Julia Cristina Gerlero M. A. por su atenta lectura de este texto y sus útiles consejos respecto para con una expresión más clara. Ha aparecido una versión en inglés de este artículo, «Indigenous Knowledge, Land Ethic and Sustainability», Electronic Journal of Australian and New Zealand History (2000), http://www.jeu.edu.au/ aff/history/articles/heyd.htm. 